\section{Extracorporeal shock wave lithotripsy as rescue for a balloon dilator trapped in the pancreatic duct}

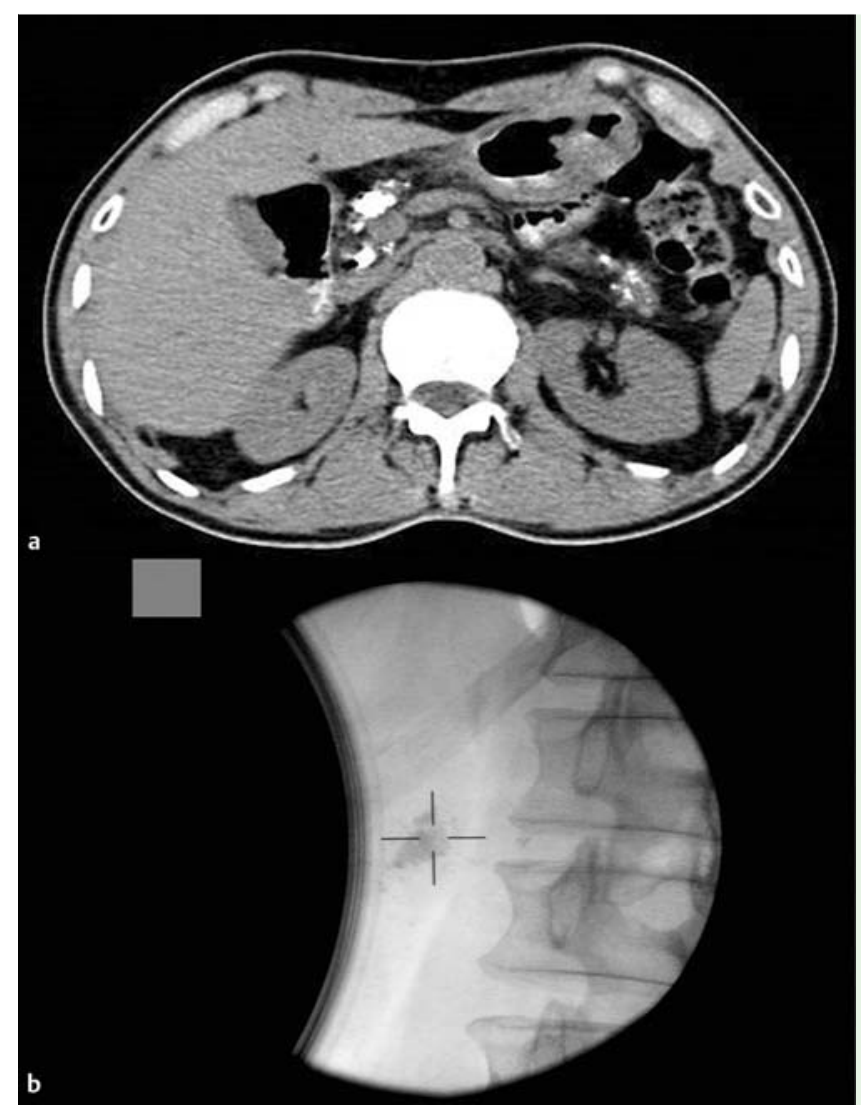

Fig. 1 a Computed tomographic scan reveals a severely dilated pancreatic duct with multiple radiopaque stones, confirming a diagnosis of chronic pancreatitis in a 71-year-old man admitted because of intermittent upper abdominal pain of 2 years' duration. b The pancreatic stones are pulverized after extracorporeal shock wave lithotripsy.

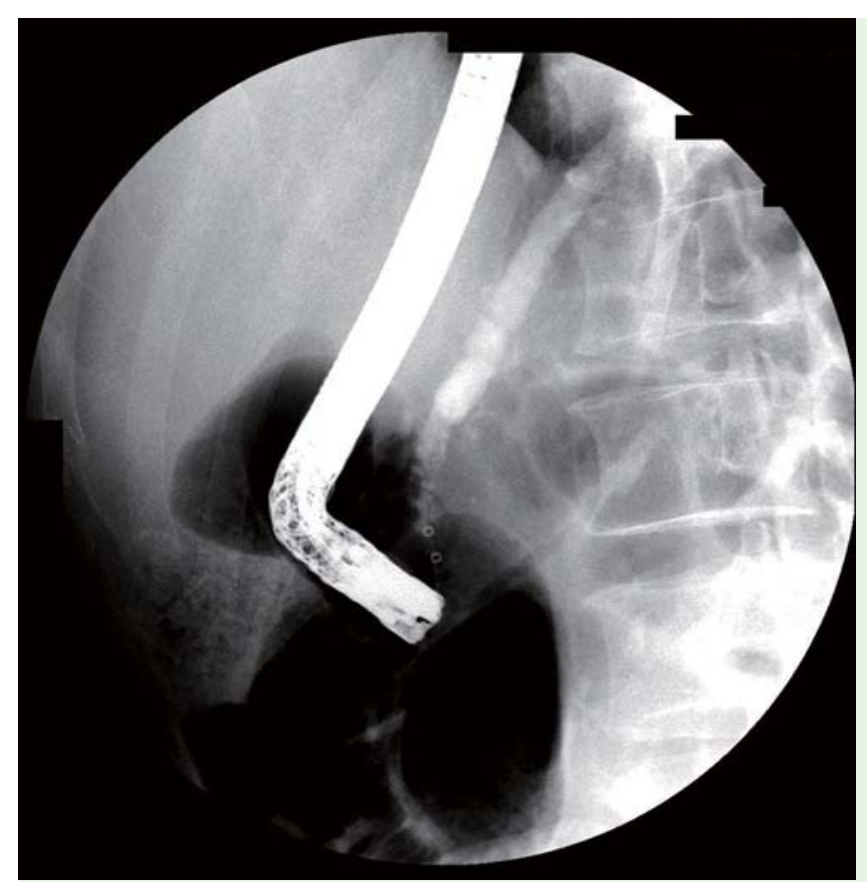

A 71-year-old man was admitted to our department because of intermittent upper abdominal pain of 2 years' duration. Computed tomography revealed a severely dilated pancreatic duct with multiple radiopaque stones, confirming a diagnosis of chronic pancreatitis ( Fig.1a). We performed extracorporeal shock wave lithotripsy (ESWL) at the pancreatic head with a third-generation lithotripter (Compact Delta II; Dornier MedTech, Wessling, Germany) to break up the stones. A total of 5000 shocks were delivered during the therapeutic session at an intensity of 6 on a scale of 1 to $6(16000 \mathrm{kV})$ and a frequency of 120 shocks per minute. A combination of flurbiprofen and remifentanil was administered via intravenous infusion for analgesia. The pancreatic stones were pulverized after ESWL ( $\bullet$ Fig. 1 b) [1]

After 48 hours, endoscopic retrograde pancreatography (ERP) was performed to remove the residual fragments. ERP showed a severe stricture at the pancreas head and a dilated proximal pancreatic duct. Following sphincterotomy, a balloon dilator with a diameter of $8 \mathrm{~mm}$ and length of $3 \mathrm{~cm}$ (Quantum TTC; WilsonCook Medical, Winston-Salem, North Carolina, USA) was used to dilate the stricture. However, the stone fragments punctured and broke the balloon. When we tried to pull out the balloon dilator, we encountered a high degree of resistance and failed to remove it.

ERP showed that the two markers on the balloon dilator were closer together than normal, indicating that the balloon was folded and trapped in the main pancreatic duct (MPD) ( Fig.2, $\bullet$ Fig.3). The plunger handle of the balloon dilator was then cut off, and the duodenoscope was withdrawn. We tried to insert a guidewire beside the distorted balloon with the duodenoscope, but it inevitably slipped into a branch of the pancreatic duct without passing the trapped balloon. The duodenoscope was again withdrawn ( $\bullet$ Video 1 ). The balloon catheter was extracted through the nasal cavity and connected to a Tuohy-Borst connector as a nasopancreatic tube ( $\bullet$ Fig. 4 ).

To release the trapped balloon, another ESWL procedure was performed to break up the impacted stones into smaller pieces, targeting the stones around the two markers ( $\mathbf{F i g}$.5). At 48 hours after the second ESWL, a second ERP was performed. A Guide Wire M (Radifocus; Terumo Corporation, Tokyo, Japan) and a 6-Fr plastic bougie dilator were inserted, but the latter could not pass through the stric- 


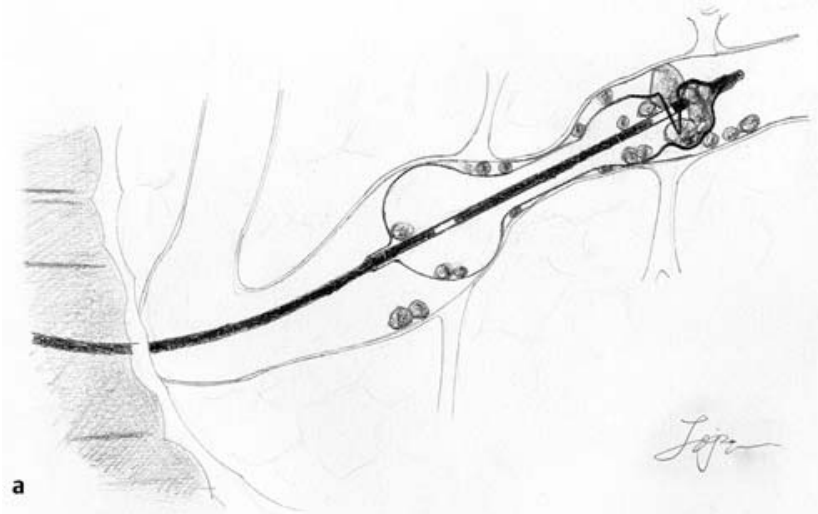

Fig. 3 The trapped balloon (illustration by J.T. ji). a The balloon has been punctured and broken by stone fragments, which then enter the balloon.

b The trapped balloon is distorted and folded after a series of endoscopic procedures.

b

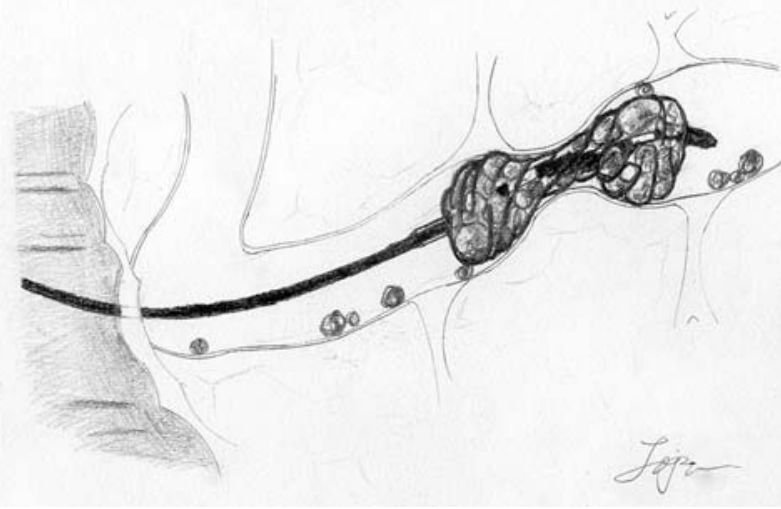

ture. The plastic bougie dilator was used as a support while the Guide Wire M was replaced with a standard guidewire, and the pancreatic duct was then dilated with a stent retriever (Soehendra; Wilson-Cook Medical) and multilevel plastic bougie dilators. Finally, a balloon dilation catheter with a diameter of $8 \mathrm{~mm}$ and length of $4 \mathrm{~cm}$ (Hurricane; Boston Scientific, Natick, Massachusetts, USA) was used to dilate the stricture. The broken, folded, and distorted balloon dilator ( $\mathbf{F i g . 6}$ ) was then pulled out ( $\bullet$ Video 2 ).

Stones of any size can cause a balloon to break, regardless of whether or not ESWL is performed first. Broken balloons are rarely trapped in the MPD. When a trapped balloon dilator cannot be withdrawn by endoscopic techniques, surgery is usually the only choice. However, the results of our case show that ESWL can be a safe and effective way to break up culprit stones into smaller pieces and release a trapped balloon [2-5].

\section{Endoscopy_UCTN_Code_CPL_1AK_2AF}

\section{Competing interests: None}
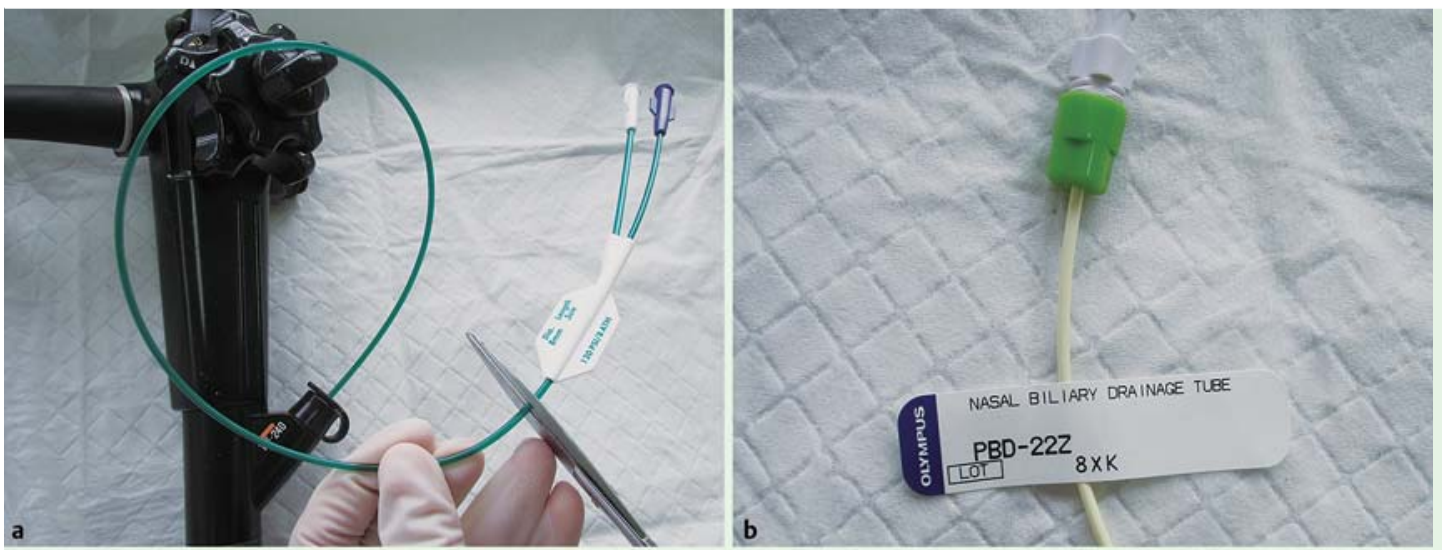

Fig. 4 Drainage system. a The handle of the balloon dilator is cut off. b Nasal biliary drainage tube. $\mathbf{c}$ The catheter is extracted through the nasal cavity and connected to the Tuohy-Borst connector as a nasopancreatic tube. $\mathbf{d}$ The nasopancreatic tube and negative pressure drainage bag are connected with
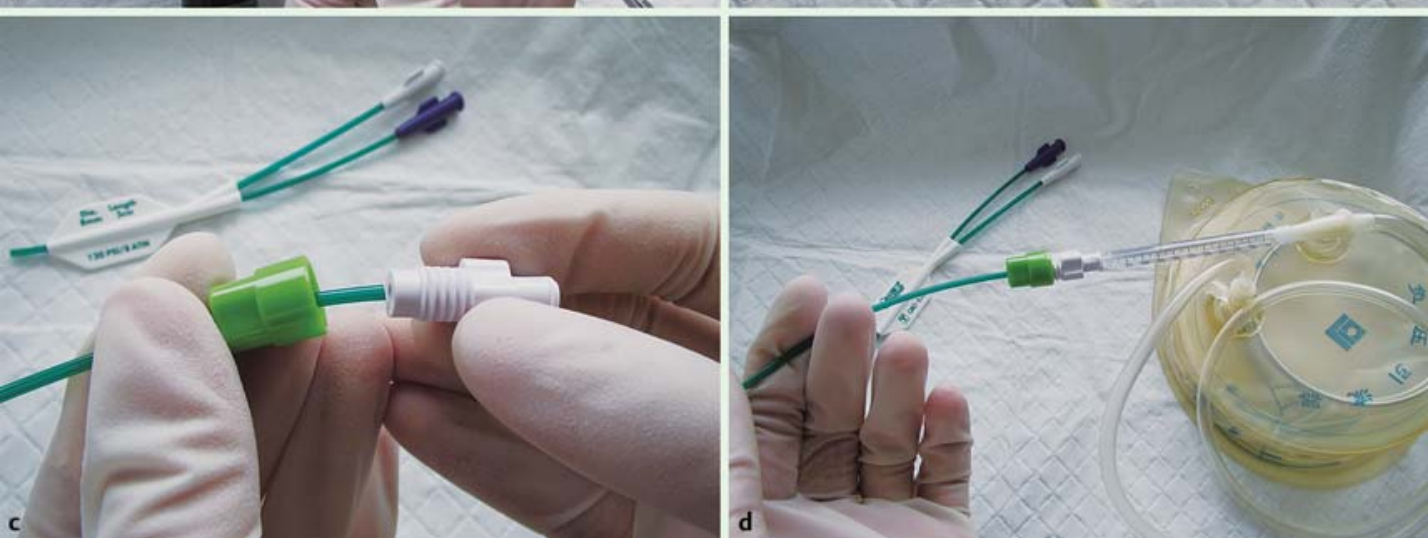
a 1 -mL syringe. 


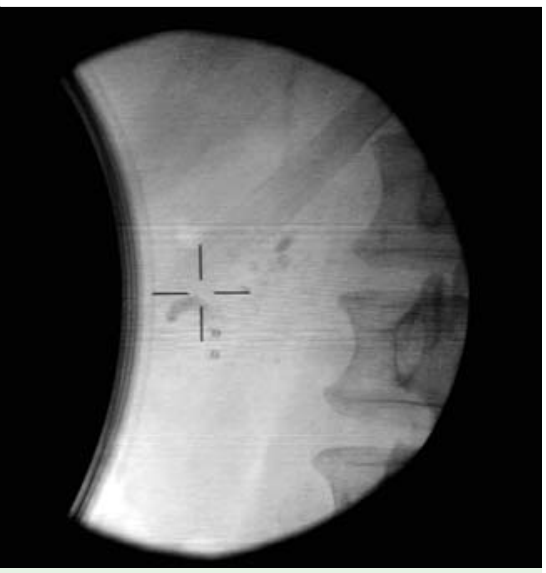

Fig. 5 Stones around the two markers are targeted during the second extracorporeal shock wave lithotripsy procedure.

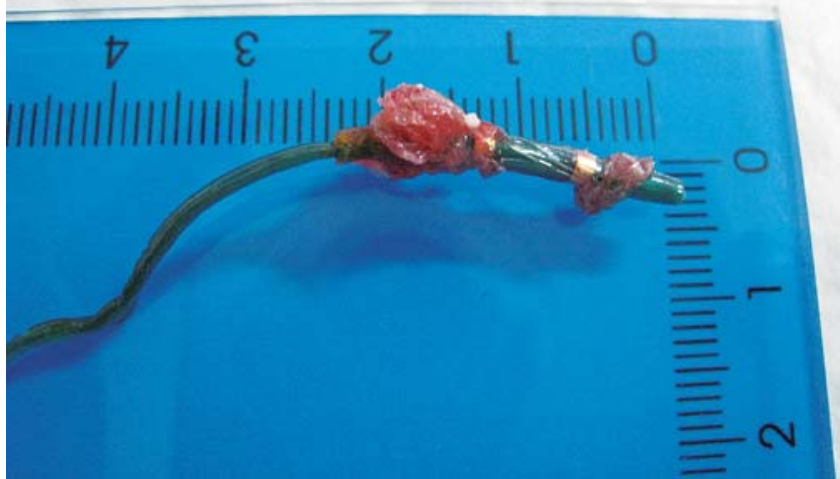

Fig. 6 The broken, folded, and distorted balloon dilator.

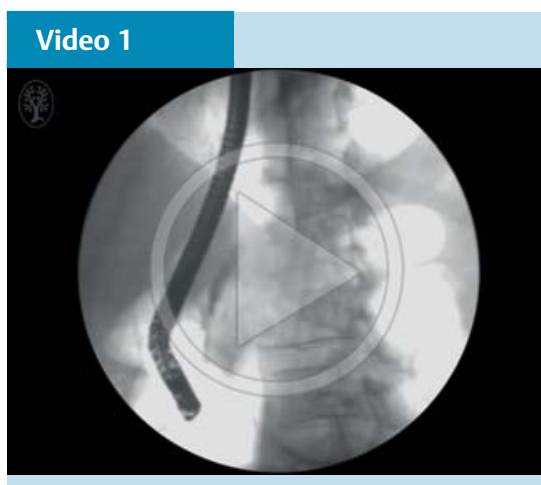

The first endoscopic retrograde pancreatography procedure.

\section{Dan Wang ${ }^{1, ~ *}$, Jun-Tao $\mathrm{ji}^{2}$, *, Duo-Wu Zou$^{2}$, Liang-Hao Hu${ }^{1}$, Zhao-Shen Li ${ }^{1}$}

${ }^{1}$ Department of Gastroenterology, Changhai Hospital, Second Military Medical University, Shanghai, China ${ }^{2}$ Digestive Endoscopy Center, Changhai Hospital, Second Military Medical University, Shanghai, China

\section{References}

1 Li BR, Liao Z, Du TT et al. Risk factors for complications of pancreatic extracorporeal shock wave lithotripsy. Endoscopy 2014; 46: $1092-1100$

2 Dumonceau JM, Delhaye M, Tringali A et al. Endoscopic treatment of chronic pancreatitis: European Society of Gastrointestinal Endoscopy (ESGE) Clinical Guideline. Endoscopy 2012; 44: 784-800

3 Maydeo A, Soehendra N, Reddy $N$ et al. Endotherapy for chronic pancreatitis with intracanalar stones. Endoscopy 2007; 39: 653 658

$4 \mathrm{Hu}$ LH, Liao Z, Li ZS. Spontaneous clearance of pancreatic stones. Clin Gastroenterol Hepatol 2013; 11: e9-e10

$5 \mathrm{Hu}$ LH, Liao Z, Li ZS. Rolling in the deep: a quaint sphere rolling in the deep pancreatic duct. Gastroenterology 2013; 145: e7-e8

Bibliography

Dol http://dx.doi.org/

10.1055/s-0034-1393668

Endoscopy 2015; 47: E604-E606

(c) Georg Thieme Verlag KG

Stuttgart · New York

ISSN 0013-726X

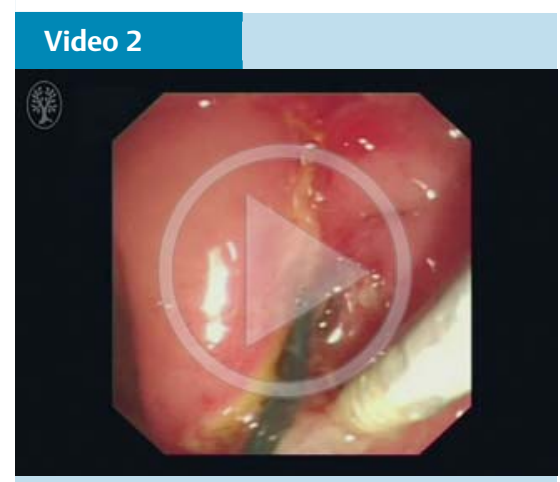

The second endoscopic retrograde pancreatography procedure.

Corresponding author

\section{Zhao-Shen Li, MD}

Department of Gastroenterology

Changhai Hospital

Second Military Medical University

168 Changhai Road

Shanghai

China

Fax: +86-21-55620081

zhaoshen-li@hotmail.com

\section{Liang-Hao Hu, MD}

Department of Gastroenterology

Changhai Hospital

Second Military Medical University

168 Changhai Road

Shanghai

China

Fax: +86-21-55620081

lianghao-hu@hotmail.com
* Dr. Wang and Dr. Ji contributed equally to this work. 